\title{
Frutose em humanos: efeitos metabólicos, utilização clínica e erros inatos associados
}

\author{
Fructose in humans: metabolic effects, clinical \\ utilization, and associated inherent errors
}

Rodrigo Crespo BARREIROS'

Grasiela BOSSOLAN²

Cleide Enoir Petean TRINDADE²

RE S U M O

Revisa-se o metabolismo da frutose e do sorbitol, suas principais indicações e conseqüências decorrentes do uso inadequado. A frutose é um importante carboidrato da dieta, sendo encontrada principalmente nas frutas e vegetais, e é produzida no organismo a partir da glicose pela via do sorbitol. A frutose é conhecida pelos erros inatos do seu metabolismo, cujas manifestações clínicas são potencialmente graves, e por seu uso como substituta da glicose na dieta de diabéticos, visto não depender da insulina para o seu metabolismo. Nos últimos anos, especialmente em países desenvolvidos, seu consumo tem aumentado acentuadamente em virtude do emprego como adoçante em produtos industrializados. Porém, o uso excessivo de frutose não é isento de efeitos adversos, representados pelo aumento de triglicerídios e de colesterol no sangue. O conhecimento dos níveis sangüíneos normais é importante tanto para estabelecer a quantidade segura a ser administrada, como para permitir avaliar doenças metabólicas associadas à frutose.

Termos de indexação: doenças metabólicas, fisiopatologia, frutose, insulina, metabolismo.

\section{A B S T R A C T}

This article reviews the metabolism of fructose and sorbitol, their main clinical indications and the consequences of inadequate use. Fructose, an important carbohydrate in the diet, is present mostly in fruits and vegetables; it can also be synthesized from glucose in the organism, through the sorbitol. Fructose is known for its metabolism's inherent errors, whose clinical manifestations are potentially serious, as well as for its use as a glucose substitute in the diabetic patients' diet, due to its metabolism not being dependent from insulin. In

\footnotetext{
1 Departamento de Medicina, Centro de Ciências Médicas e Biológicas, Faculdade de Medicina de Sorocaba, Pontifícia Universidade Católica de São Paulo. Praça José Ermírio de Moraes, 290, Vergueiro, 18030-230, Sorocaba, SP, Brasil. Correspondência para/Correspondence to: R.C. BARREIROS. E-mail:<rodcbarr@splicenet.com.br>.

2 Departamento de Pediatria, Faculdade de Medicina, Universidade Estadual Paulista de Botucatu. Botucatu, SP, Brasil.
} 
the last years, especially in developed countries, the consumption of fructose has increased considerably, due to its use as a sweetener in industrialized foods. However, adverse side-effects may occur with the excessive ingestion of fructose, such as the increase in blood's triglycerides and cholesterol. Therefore, to know which are the patients' normal blood levels is quite important for establishing the safe amount of fructose to be prescribed, as well as for allowing the screening of metabolism diseases associated with fructose.

Indexing terms: metabolic diseases, physiopathology, fructose, insulin, metabolism.

\section{N T R O D U Ç Ã O}

A frutose é um importante carboidrato encontrado no organismo humano e na maioria das plantas, tendo sido isolada pela primeira vez em 1847 a partir da cana-de-açúcar'. Seu nome é originário da palavra latina fructus, já que as frutas são uma importante fonte de frutose.

Como componente de frutas e outros vegetais, é ingerida regularmente com a dieta. Também é sintetizada no organismo a partir da glicose, via sorbitol, e esse processo se relaciona com a manutenção do equilíbrio óxido-redutivo.

A frutose é mais conhecida por erros inatos associados ao seu metabolismo que podem ter conseqüências clínicas importantes e também como possível substituta da glicose na dieta dos diabéticos, pelo fato de possuir estrutura química semelhante à da glicose, mas não necessitar da insulina para o seu metabolismo. Entretanto, seus efeitos metabólicos, principalmente os relacionados ao metabolismo lipídico e toda uma rede de metabólitos, são bem menos divulgados.

Considerando que, nos últimos anos, especialmente em países desenvolvidos, a ingestão de frutose vem aumentando acentuadamente, em decorrência do maior consumo de produtos industrializados contendo frutose e sorbitol como adoçantes, é importante conhecer seus aspectos metabólicos, bem como os efeitos associados ao seu uso inadequado? .

O objetivo deste artigo foi reunir as mais recentes informações a respeito do metabolismo da frutose, assim como as principais indicações para o seu uso e as conseqüências do uso abusivo.
C AR ACTERÍSTICAS FÍ S I C O-Q U ÍM I C A S

A frutose é considerada um açúcar, sendo um composto sólido, incolor, cristalino e solúvel em água. O termo "açúcar" deriva do sânscrito çarkara, que significa grão de areia. O termo em sânscrito deu origem ao grego sakkaron, ao latim saccharum e ao árabe sukkar. A palavra portuguesa açúcar tem origem árabe. Os açúcares ${ }^{3}$ são carboidratos e apresentam carbono, hidrogênio e oxigênio na sua composição em uma proporção de $1: 2: 1$.

A frutose é um monossacarídio, pois é composta por seis átomos de carbono unidos em ligações covalentes simples, apresentando grupamentos hidroxila, formados por hidrogênio e oxigênio e um grupamento carbonila, formado por ligação dupla entre o carbono e o oxigênio. A posição desse grupamento é que determinará, após a hidrólise do monossacarídio, se ele dará origem à cetona ou aldeído. A frutose, contendo o grupamento carbonila no final da cadeia, quando hidrolisada, fornecerá cetona, e será denominada cetohexose. A glicose, por sua vez, quando hidrolisada, dará origem a aldeído, sendo chamada de aldohexose ${ }^{4}$.

A oxidação dos carboidratos é a principal via metabólica produtora de energia para a maioria das células não fotossintéticas, fornecendo um grama de frutose $16 \mathrm{~kJ}$ de energia ${ }^{4}$.

\section{Fontes de frutose}

A frutose, também conhecida como levulose, além de ser encontrada sob forma isolada na natureza, é constituinte da sacarose ( $\beta$-D-Frutofuranosil $\alpha$-D-glicopiranosida) e de 
outros polímeros denominados fructans ou inulina ${ }^{5}$. A inulina está presente na chicória, batata-doce e alcachofra de Jerusalém. A frutose é o monossacarídio predominante em várias frutas, incluindo maçãs, laranjas e melões. Os vegetais podem conter de $1 \%$ a $2 \%$ de seu peso na forma de frutose livre e mais $3 \%$ de frutose sob a forma de sacarose ${ }^{6}$.

A frutose pode ser encontrada como rafinose, que é um trissacarídio, e também como estaquiose, que é um tetrassacarídio, em algumas leguminosas como a soja, lentilha, ervilha e feijão. Essas formas não são absorvidas pelo intestino humano, sendo fermentadas pelas bactérias no intestino grosso, gerando gases que acompanham a digestão dessas leguminosas ${ }^{7}$.

Na década de 70, a separação da frutose e da glicose a partir de soluções compostas por açúcares invertidos foi conseguida por meio da cromatografia de troca iônica. A utilização desse método aumentou a produção de frutose pura e diminuiu drasticamente o seu preço. Ao mesmo tempo, o isolamento de isomerases capazes de transformar a D-glicose em D-frutose levou à introdução comercial de xaropes derivados de amido, ricos em frutose 1 .

A frutose vem sendo empregada como adoçante de bebidas e frutas industrializadas, constituindo de $4,0 \%$ a $8,0 \%$ de seu peso em decorrência de algumas características, como a maior solubilidade em soluções aquosas e pelo fato de ser mais doce, cerca de 1,7 vez mais que a sacarose. Alimentos produzidos em confeitarias têm, em média, 1,0\% a 2,0\% de frutose, porém se esses alimentos apresentarem frutas na composição, a quantidade de frutose pode aumentar para cerca de 11,0\%. O mel fornece a maior concentração de frutose ( $42,4 \%$ do peso), sendo considerado um adoçante natural ${ }^{7}$.

Quadro 1. Açúcares simples em alguns alimentos.

\begin{tabular}{|c|c|c|c|c|}
\hline Alimentos & Frutose & Glicose & Sacarose & Sorbitol \\
\hline \multicolumn{5}{|l|}{ Frutas } \\
\hline Maçã & $6-8,0$ & $1-4,0$ & $1-5$ & $0,2-1,0$ \\
\hline Suco de maçã & $6-8,0$ & $1-4,0$ & 4 & $0,3-1,0$ \\
\hline Banana & $2-4,0$ & $3-6,0$ & $6-14$ & $?$ \\
\hline Cereja & $5-7,0$ & $5-7,0$ & 0,2 & $1,4-2,1$ \\
\hline Uva & $5-7,0$ & $5-7,0$ & 0,5 & 0,2 \\
\hline Laranja & $2-3,0$ & $2-3,0$ & $4-7$ & - \\
\hline Suco de laranja & $2-6,0$ & $2-6,0$ & $2-4$ & - \\
\hline Pêra & $5-9,0$ & $1-2,0$ & $1-2$ & $1,2-3,5$ \\
\hline Suco de pêra & $5-9,0$ & $1-2,0$ & $1-2$ & $1,1-2,6$ \\
\hline Ameixa & $1-4,0$ & $2-5,0$ & $1-5$ & $0,3-2,8$ \\
\hline Morango & $1-3,0$ & $1-3,0$ & $1-2$ & $<0,1$ \\
\hline Melão & $2-4,0$ & $1-2,0$ & $1-5$ & $1,0-5,0$ \\
\hline Tomate & $1-1,5$ & $1-1,5$ & $<0,1$ & $<0,1$ \\
\hline Mel & 41 & 34 & 2 & - \\
\hline \multicolumn{5}{|c|}{ Hortaliças e Vegetais } \\
\hline Aspargos & 1,4 & 1,0 & 0,3 & - \\
\hline Feijão & $1-1,5$ & $0,5-1,0$ & 0,6 & - \\
\hline Repolho & 1,6 & 1,7 & 0,4 & - \\
\hline Cenoura & 1,0 & 1,0 & 4,0 & - \\
\hline Alho & 1,5 & 1,0 & $1-1,5$ & - \\
\hline Alface & 0,6 & 0,5 & 0,1 & - \\
\hline Cebola & 1,0 & 2,0 & 1,0 & - \\
\hline Ervilha & $<0,1$ & $<0,1$ & $1-5,0$ & - \\
\hline Batata & $<0,1$ & $<0,1$ & $<0,1$ & - \\
\hline
\end{tabular}

Fonte: Hallfrisch ${ }^{7}$ 
A frutose pode ainda ser produzida a partir do sorbitol. Isso ocorre quando esse poliól é oxidado em uma reação mediada pela enzima sorbitol-desidrogenase. O sorbitol está presente em várias plantas, em particular na família das Rosáceas (maçãs, peras, cerejas, ameixas e abricós), geralmente associado à frutose (Quadro 1). Outras fontes de sorbitol são doces, balas de menta, goma de mascar, alimentos e sucos dietéticos 5 .

\section{Consumo de frutose e sorbitol}

Na dieta humana ocidental, os carboidratos correspondem de 200 a 300g/dia ou a cerca de $40 \%$ a $50 \%$ da energia ingerida. A ingestão diária de frutose livre (excluindo a sacarose), nos EUA, é de cerca de $16 \mathrm{~g} / \mathrm{dia}$, com um percentil 90 de $31 \mathrm{~g} /$ dia. Caso haja ingestão de xaropes de milho, ricos em frutose, o consumo pode atingir 60 a $100 \mathrm{~g} /$ dia e até $150 \mathrm{~g} /$ dia se somada a frutose proveniente da sacarose ${ }^{5}$.

A ingestão de frutose no Brasil não está bem estabelecida. Estima-se um consumo médio de cerca de 4,34g/dia de frutose livre, originária de frutas, doces, hortaliças e outros vegetais. A quantidade de frutose provinda da sacarose é de aproximadamente $27,5 \mathrm{~g} / \mathrm{dia}$. Essa estimativa foi baseada em dados estatísticos de consumo de produtos alimentares fornecidos pelo Instituto Brasileiro de Geografia e Estatística, utilizando-se como fonte as pesquisas sobre orçamentos familiares, realizadas entre outubro de 1995 e setembro de $1996^{8}$.

Estudos mostraram que a dieta do brasileiro vem sendo modificada com uma tendência para redução do consumo de leguminosas, hortaliças e frutas e aumento do consumo de açúcares simples e conseqüentemente de frutose, principalmente a proveniente da sacarose ${ }^{9}$. Essa mudança nos hábitos alimentares poderá repercutir no metabolismo lipídico e ocasionar aumento dos lipídios totais e triglicerídios na circulação.

\section{Absorção intestinal}

A frutose é absorvida através de dois mecanismos distintos, sendo o primeiro um transporte facilitado, independente da glicose, com baixa capacidade, e, o segundo, um co-transporte, dependente da glicose e com alta capacidade ${ }^{10}$.

A difusão facilitada é mediada pela proteína GLUT, que no caso da frutose é a GLUT5. Esse transportador tem alta afinidade com a frutose e pouca afinidade com a glicose. Seu papel primário é o de atuar no transporte da frutose da dieta e sua ação não depende da estimulação pela insulina ${ }^{11}$.

A absorção da frutose aumenta quando ela é ingerida sob a forma de sacarose ou quando misturada com a glicose, pois, durante a absorção da glicose, ocorre a abertura de pequenas junções, com movimento de fluido luminal por meio de vias paracelulares. Através desse movimento, pequenos solutos, incluindo a frutose, se movem passivamente, aumentando em $29 \%$ a absorção da frutose quando associada a soluções com glicose $\mathrm{e}^{12}$.

O sorbitol é incompletamente absorvido e, quando oferecido simultaneamente com a frutose, compete com ela pelo sítio de absorção ${ }^{13}$.

Após a absorção, a frutose sai do enterócito pela membrana basolateral atingindo o sangue da veia porta que a transporta até o fígado ${ }^{11}$. Esse transporte é mediado pela GLUT 2, que está situada na membrana basolateral do enterócito.

\section{Metabolismo da frutose}

A frutose é primariamente metabolizada no fígado, apesar de o intestino e os rins possuírem enzimas necessárias para o seu catabolismo. Sua rápida entrada no hepatócito é mediada também pela GLUT 2, não havendo gasto de energia ou necessidade do estímulo pela insulina. No hepatócito, a frutose é rapidamente fosforilada no carbono 1, em uma reação mediada pela frutoquinase ou cetoquinase, ou 
no carbono 6, em uma reação mediada pela hexoquinase. A maior parte da frutose é fosforilada no carbono 1 , pois a hexoquinase tem maior afinidade com a glicose ${ }^{7}$.

A frutose-1 fosfato é cindida em duas trioses, diidroxiacetona e gliceraldeído-fosfato, em uma reação mediada pela aldolase B. Essas duas trioses poderão seguir três caminhos distintos, com finalidades diferentes: participar da via glicolítica fornecendo piruvato e liberando energia, ser reduzidas até glicerol, necessário para a síntese de triacilgliceróis, fosfolipídios e outros lipídios e, finalmente, ser condensadas até formar a frutose1,6-difosfato e, a partir dessa, formar glicose ou glicogênio. Dessa forma, dará origem ao piruvato, lipídios e ao glicogênio. Na Figura 1, estão apresentadas as vias metabólicas da frutose e a inter-relação com o metabolismo lipídico e glicídico.

O controle do metabolismo da frutose está relacionado com um derivado fosforilado da frutose. Trata-se da frutose-2,6-difosfato, descoberta em 1980 por Hers e colaboradores e que está presente em todos os tecidos dos mamíferos, exceto nos eritrócitos maduros, sendo o mais potente efetor da fosfofrutoquinase ${ }^{14}$. A frutose-2,6 difosfato desempenha importante papel na regulação da glicólise em vários tipos de células e também controla a gliconeogênese nas células que possuem essa habilidade ${ }^{14}$.

A frutose pode ser produzida a partir do sorbitol, através da via do sorbitol, que é composta por duas reações em que a forma não fosforilada da glicose é convertida em frutose tendo como intermediário o sorbitol. A primeira reação necessita de aldolase-redutase e oxida NADPH, enquanto a segunda reação reduz NAD+e depende da cetose-redutase ${ }^{7}$. A frutose, pela via do sorbitol, é produzida na próstata e vesícula seminal, a partir da glicose, tendo papel importante no suprimento de energia e mobilidade do espermatozóide (Figura 1). A concentração seminal de frutose é um indicador do tamanho e da capacidade secretora da vesícula seminal. O seu metabolismo é feito através da glicólise via Embden-Meyerhof'.

\section{Efeitos metabólicos da frutose em seres humanos}

Na prática nutricional corrente, a ingestão moderada de frutose presente nos alimentos naturais tem efeitos benéficos a partir da sua utilização como elemento energético. Entretanto, é importante salientar o crescente aumento no seu uso como adoçante em produtos industrializados e que, portanto, a possibilidade de produzir lipídios por meio dos compostos intermediários como o glicerol e o gliceraldeído, levando ao aumento dos lipídios sangüíneos, deve ser considerada, principalmente na população com risco de problemas cardíacos.

Há uma nítida inter-relação entre o metabolismo da frutose e o da glicose. A frutose administrada oralmente ou por via endovenosa é captada pelas células do fígado, onde é convertida em glicose e principalmente em glicogênio. Apesar da estimulação da gliconeogênese, a administração de frutose pura produz apenas pequenos aumentos da glicemia ${ }^{15}$.

A frutose, além de ser uma fonte de energia em substituição a outros carboidratos da dieta, apresenta, também, um efeito catalítico, isto é, pode estimular a translocação da glicoquinase para fora do núcleo do hepatócito. A glicoquinase translocada será responsável pela fosforilação da glicose, uma etapa determinante do metabolismo hepático da glicose. Adultos normais apresentam aumento significante na tolerância à glicose quando doses baixas (catalíticas) de frutose são adicionadas ao montante de glicose durante o teste de tolerância à glicose ${ }^{16}$.

Quanto à produção de lipídios a partir da frutose, estudos em animais e em seres humanos demostraram aumento nos triglicerídios após a ingestão de dietas contendo frutose quando comparadas às dietas com carboidratos mais complexos e outros açúcares. Há aumento da síntese de gordura em detrimento da gliconeogênese, e esse aumento ocorre pela maior síntese hepática de glicerol e de ácidos graxos, 1,4 a 18,9 
vezes, quando comparamos com a glicose. 0 aumento da atividade das enzimas lipogênicas no fígado resulta em maior síntese de lipídios, e, como conseqüência, níveis mais elevados de lipídios totais na circulação e de lipoproteínas de muito baixa densidade (VLDL) .

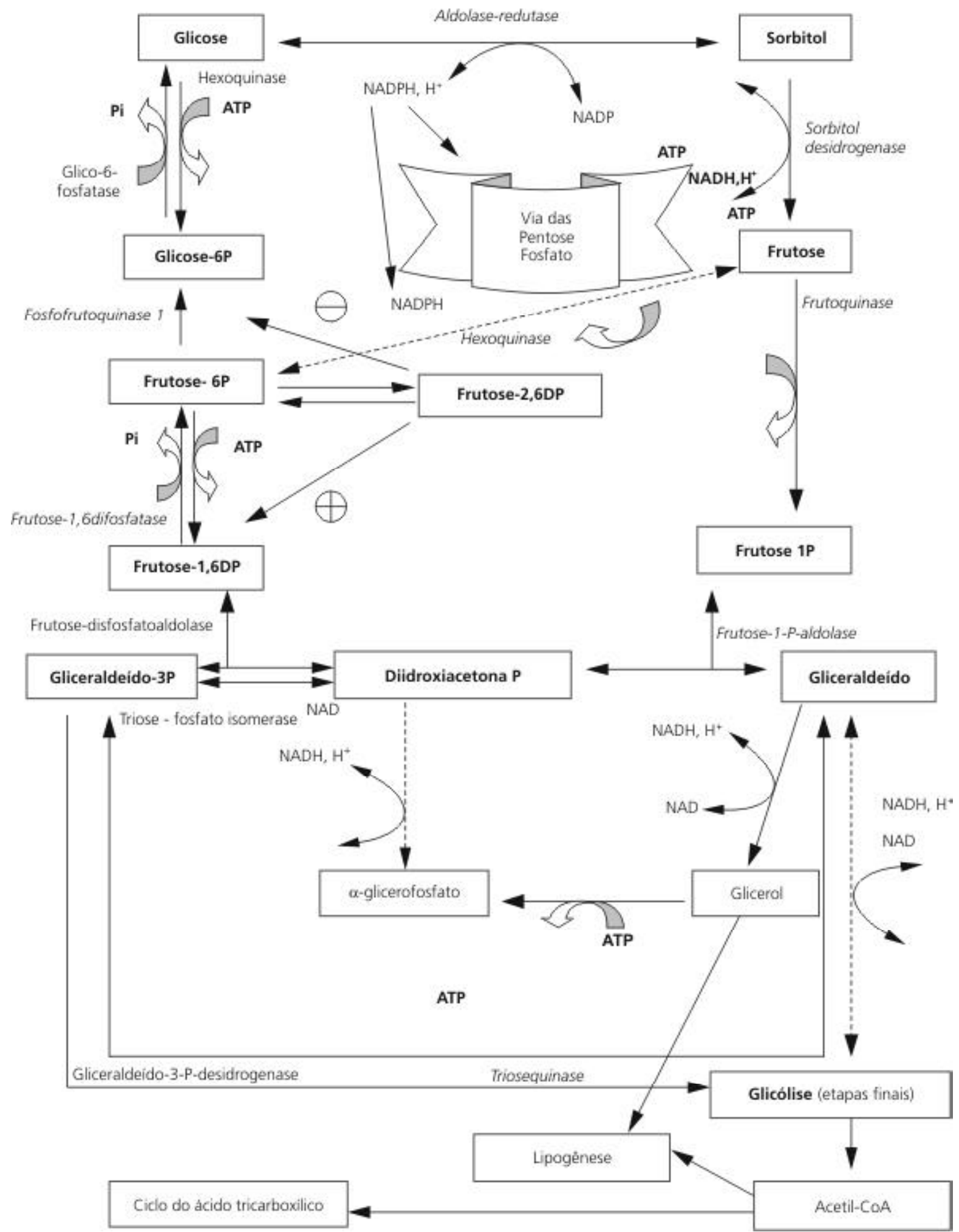

Figura 1. Vias metabólicas da frutose e inter-relação com os metabolismos lipídico e glicídico (adaptado de Hallfrisch)7. 
Em seres humanos, alguns grupos são mais susceptíveis a apresentar hipertrigliceridemia após a ingestão de frutose, como mulheres pós-menopausa, homens hiperinsulinêmicos e diabéticos tipo 2. Outro fator importante é a ação conjunta de gordura e frutose, que leva a um efeito sinérgico, resultando em um clareamento mais lento de triglicerídios ${ }^{7}$.

Outros aspectos do metabolismo da frutose foram revisados em estudos por Reiser et al. ${ }^{17}$, tendo sido relatado aumento nos níveis de ácido úrico plasmático após o consumo de frutose presente na dieta, sendo mais propensos os pacientes com hipertensão arterial. A hiperuricemia observada é provavelmente decorrente de aumento do catabolismo dos nucleotídeos ou de aumento na síntese de purinas. Evidências confirmam as duas hipóteses, pois biópsias hepáticas mostraram diminuição no conteúdo de nucleotídeos após a infusão de frutose e também aumento na incorporação de glicina radioativa no trato urinário, indicando aumento na síntese de purinas.

A frutose na dieta pode aumentar significativamente os níveis de lactato, devido ao incremento na atividade da frutoquinase, que supera a capacidade de ação da fosfo-frutoquinase e desvia o metabolismo para a glicólise em detrimento da gliconeogênese. Os níveis de lactato decorrentes da frutose da dieta são menores que os encontrados após a infusão venosa de frutose ${ }^{7}$.

\section{Utilização clínica da frutose}

O uso de frutose na dieta de diabéticos foi preconizado desde 1893, quando Minkowski observou a independência da frutose em relação à insulina para a sua metabolização. Vários estudos posteriores reforçaram a idéia de que a frutose, assim como o sorbitol, seriam benéficos na dieta de diabéticos ${ }^{18}$.

Existem alguns argumentos a favor da utilização da frutose em diabéticos: independência em relação à insulina no transporte e distribuição de frutose e do sorbitol até a etapa inicial do metabolismo; absorção mais lenta da frutose e do sorbitol em relação à glicose; aumentos mínimos e transitórios da glicemia após a absorção de frutose e sorbitol; o fato de a síntese de glicogênio em humanos diabéticos acontecer de maneira adequada após a ingestão de frutose e sorbitol, independentemente do nível de glicose sangüínea; e o fato de a frutose e o sorbitol terem efeito anti-cetogênico'.

Não existe consenso em relação ao uso desses açúcares entre os especialistas em diabetes. Os autores contrários ao uso consideram que o emprego descontrolado da frutose e do sorbitol resultaria em glicose e seu polímero, o glicogênio, e, conseqüentemente, aumento na glicemia. A tendência atual é aceitar a frutose e o sorbitol como adoçantes, uma vez que dietas com restrição de carboidratos não são bem aceitas pelos diabéticos. Portanto, pela pouca interferência nos níveis de glicose sangüínea, torna-se uma alternativa para diabéticos que apreciam alimentos doces. A frutose presente na dieta produz menor aumento na glicemia quando comparada a quantidades isocalóricas de sacarose e de amido, sendo essa uma vantagem da frutose como adoçante na dieta dos diabéticos. Quando empregada em grande quantidade, o dobro do normal, ou $20 \%$ das calorias ingeridas, podem ocorrer efeitos colaterais como o aumento nos níveis de colesterol. O consumo de grandes quantidades deve ser evitado em pessoas com dislipidemia, porém diabéticos podem consumir frutas e vegetais sem restrição e ingerir com moderação alimentos contendo frutose como adoçante ${ }^{19}$.

De acordo com o consenso de 2002 da Associação Norte-Americana de Endocrinologia (AACE), o consumo de carboidratos recomendado para pacientes diabéticos deve corresponder de $55 \%$ a $60 \%$ do total de calorias ingeridas e o total de carboidratos, mais do que a fonte, parece ser o fator crítico que determina o efeito na glicemia ${ }^{20}$.

A frutose também foi utilizada na prevenção e no tratamento da hipoglicemia neonatal. 
A justificativa para a sua utilização decorreu do fato de não provocar efeito rebote de hipoglicemia, ao contrário da terapia com glicose. O seu uso foi desestimulado em decorrência dos eventuais efeitos colaterais associados ao uso da frutose, como o maior consumo de ATP, aumento de lactato e pela possibilidade de a criança ser portadora de erro inato associado à frutose ${ }^{21}$.

A frutose também tem sido utilizada como constituinte de soluções em nutrição parenteral, substituindo parcialmente ou totalmente a glicose. É considerada menos irritante para as veias, não interfere na glicemia do paciente, independente da insulina para o seu metabolismo, está associada a um menor risco de hipoglicemia após a interrupção abrupta e tem efeito poupador de proteínas ${ }^{1}$. A infusão rápida, porém, pode resultar em acúmulo de frutose-1-fosfato no fígado, rins e trato gastrintestinal com depleção intracelular de ATP dos nucleotídeos de adenina e do fósforo inorgânico. Essas mudanças estão diretamente relacionadas à dose e à velocidade de infusão'.

Outros efeitos associados à infusão venosa são a acidose láctica e a hiperuricemia. Estudos estabeleceram que a elevação plasmática e urinária de ácido úrico em humanos ocorre devido à rápida degradação do ATP hepático usado na reação catalisada pela frutoquinase ${ }^{7}$.

Experimentos com ratos usando infusão de frutose por via parenteral demonstraram que a concentração de ATP caía em aproximadamente $40 \%$ após dois minutos, seguindo-se de aumento equivalente de AMP e ADP. A diminuição de ATP e $P_{i}$ é seguida por catabolismo até os produtos finais, tendo como conseqüência elevação no ácido úrico e na uridina plasmática e urinária².

O uso de frutose também foi sugerido no manuseio dietético da diarréia intratável da infância, como fonte alternativa de carboidrato para crianças incapazes de tolerar os polímeros de glicose ${ }^{22}$.

A frutose foi também utilizada no tratamento da intoxicação alcoólica aguda. Estudos indicaram que $500 \mathrm{~mL}$ de frutose a $40 \%$, ofertados em um período de 30 minutos, aumentavam em cerca de $25 \%$ a taxa de declínio dos níveis de etanol sangüíneo. As doses recomendadas variaram de um a três litros de solução de frutose a 10\%. Contudo, observou-se a presença de acidose láctica potencialmente grave. A falta de pesquisas controladas e de outras evidências convincentes contra-indicam a sua utilização de rotina na intoxicação alcoólica aguda ${ }^{23}$.

Um derivado fosforilado da frutose vem sendo investigado como agente neuroprotetor na encefalopatia hipóxico-isquêmica: a frutose-1,6-difosfato. A administração desse composto, em ratos, reduz de forma significativa a lesão histológica cerebral. A atividade estabilizadora da frutose-1,6-difosfato acontece pela menor depleção de ATP através do aumento da glicólise, prevenindo a formação de radicais oxigênio-reativos e mantendo a concentração de $\mathrm{Ca}^{++}$e $\mathrm{Na}^{+}$ em níveis normais ${ }^{24}$. Outras utilizações possíveis da frutose-1,6-difosfato referem-se à melhora do desempenho cardíaco após cirurgia de colocação de marcapasso, na insuficiência cardíaca, no choque séptico e na lesão aguda pulmonar ${ }^{25}$.

\section{Concentrações de frutose no sangue}

Não existe consenso na literatura em relação aos níveis sangüíneos de frutose considerados normais em seres humanos adultos. Os valores encontrados variam, dependendo, em grande parte, do método de determinação. O mesmo pode ser dito em relação aos valores normais de sorbitol26.

Para o completo entendimento do papel da frutose e do sorbitol no organismo humano e também para que a utilização clínica desses compostos seja segura, é necessário ter conhecimento dos níveis normais no ser humano. Portanto, há a necessidade de utilização de métodos precisos e sensíveis. A dosagem desses açúcares nos fluidos orgânicos tem sido dificultada pelas baixas concentrações. Em métodos quantitativos de cromatografia gasosa, a análise 
é prejudicada pelas altas concentrações de D-glicose nas amostras. A concentração normal de frutose nos fluidos orgânicos ainda não foi definida, o que pode ser evidenciado pela grande variação em seus níveis relatada na literatura ${ }^{26}$.

Em pesquisa realizada na Faculdade de Medicina de Botucatu da Universidade Estadual Paulista (Unesp), no período de julho de 1999 a março de 2000, com o objetivo de determinar as concentrações de frutose em mães e em seus recém-nascidos e avaliar as relações maternofetais, foram determinados os níveis de frutose em sangue de cordão de recém-nascidos a termo, com pesos adequados para a idade gestacional, e em suas mães imediatamente após o parto. As determinações foram efetuadas por cromatografia líquida de alta resolução. Concluiu-se que os níveis maternos, $2,40 \pm 0,52 \mathrm{mg} / \mathrm{dL}$, foram significativamente menores do que os do cordão, $3,10 \pm 0,91 \mathrm{mg} / \mathrm{dL}$, sugerindo que há passagem placentária e possível produção de frutose pela placenta, pela via do sorbitol, como já demonstrado em humanos por Shah et al. ${ }^{27}$.

Para avaliar o comportamento após o nascimento, foram comparados os níveis de frutose do cordão com os do recém-nascido com 48 horas de vida, amamentado exclusivamente ao seio materno. O sangue foi coletado 20 minutos após o início da mamada. A concentração média em recém-nascidos com 48 horas de vida foi de $4,15 \pm 0,75 \mathrm{mg} / \mathrm{dL}$, significativamente maior do que os níveis observados ao nascimento ${ }^{28,29}$.

\section{Frutose e erros inatos do metabolismo}

Na prática clínica a frutose pode estar associada a erros inatos do metabolismo. Tais erros têm baixa incidência e os sintomas, quando presentes, são inespecíficos. As principais alterações associadas a esses distúrbios são hipoglicemia e acidose metabólica. Os erros inatos ligados à frutose são: frutosúria essencial, intolerância hereditária à frutose e deficiência da frutose-1,6-difosfatase, que serão abordados a seguir.

\section{Frutosúria essencial}

A frutosúria essencial é uma anomalia sem sintomas ou sinais visíveis. Foi descrita independentemente e simultaneamente por dois pesquisadores, Czapek e Zimmer, em $1876^{30}$. Acomete predominantemente indivíduos ${ }^{31}$ de origem judaica e sua incidência é de 1:120.000. Essa anomalia é transmitida através de herança autossômica recessiva. A frutosúria essencial é resultado da deficiência da enzima frutoquinase, acarretando uma supressão do metabolismo da frutose através da via frutose-1-fosfato, que é a via preferencial. Conseqüentemente, a frutose é parcialmente metabolizada através da conversão em frutose-6-fosfato e parcialmente excretada pela urina ${ }^{32}$. Os níveis elevados de frutose não acarretam conseqüências metabólicas e os pacientes são assintomáticos.

\section{Intolerância hereditária à frutose}

O primeiro relato de intolerância hereditária à frutose foi efetuado por Chambers e Pratt, em 1956. Seu reconhecimento como erro inato do metabolismo foi feito por Froesch et al. ${ }^{31}$, que descobriram, em 1976, que a administração de frutose em sujeitos afetados provocava grave hipoglicemia.

A intolerância hereditária à frutose tem como defeito enzimático primário a ausência aldolase $B$, responsável pela clivagem da frutose-1-fosfato. Todos os sinais e sintomas são decorrentes do acúmulo da frutose-1-fosfato, da diminuição do nível de fósforo inorgânico intracelular, do desarranjo no potencial de fosfato e das inibições enzimáticas secundárias ao acúmulo de frutose-1-fosfato, devido à inibição da fosforilação da frutose pela frutoquinase. Conseqüentemente, há o aumento de frutose no sangue e eliminação pela urina, o bloqueio da atividade da fosforilase e frutose-1,6-difosfato-aldolase, que acarreta diminuição na formação de glicose e de glicogênio, e interrupção da gliconeogênese. Os bloqueios enzimáticos 
envolvendo a fosforilase e a frutose-1,6- difosfatase explicam o aparecimento da hipoglicemia persistente. Os outros sintomas, como náuseas e vômitos, são explicados pelo acúmulo de frutose-1-fosfato e pelo desarranjo do metabolismo do fosfato e da energia na mucosa intestinal. As alterações promovendo o acúmulo de frutose-1-fosfato e alteração do metabolismo de fosfato provocam, nos rins, perda da capacidade de acidificação urinária e da reabsorção tubular de fosfato pelos túbulos ${ }^{31}$.

A forma de transmissão dessa doença é a herança autossômica recessiva, sendo os pais, via de regra, normais. A incidência de 1:40.000 é igual em ambos os sexos e tem apresentação variável. Alguns pacientes são extremamente sensíveis à frutose, enquanto outros podem tolerar quantidades moderadas. A atividade da aldolase B pode variar de indetectável até 15\% a 30\% do normal ${ }^{30}$. As manifestações dependem da introdução de alimentos que contenham frutose. Nas crianças amamentadas exclusivamente ao seio materno a sintomatologia inicia-se quando a criança passa a receber alimentos com frutose, 0 que ocorre normalmente por volta do sexto mês de vida. A criança apresenta náuseas, vômitos e hipoglicemia após a ingestão de alimentos com sacarose ou frutose, geralmente suco de laranja. As crianças com intolerância hereditária à frutose que sobrevivem até os seis primeiros meses de vida têm prognóstico melhor, uma vez que nessa idade tendem a recusar alimentos que contenham frutose.

O diagnóstico deve ser suspeitado em crianças que apresentam vômitos sem causa aparente. A investigação diagnóstica inclui a pesquisa de frutose na urina, dosagem de fosfato inorgânico sérico e de glicose sangüínea após a ingestão de substâncias contendo frutose. O teste de tolerância à frutose pode ser realizado através

Quadro 2. Dieta sem frutose - alimentos permitidos e alimentos proibidos.

\begin{tabular}{|c|c|c|}
\hline Alimentos & Permitidos & Proibidos \\
\hline Laticínios & $\begin{array}{l}\text { Leite de vaca sem açúcar, iogurte, queijo branco } \\
\text { sem açúcar, queijos naturais }\end{array}$ & logurte com frutas \\
\hline Produtos de origem animal & Carne, peixes, aves, ovos & Embutidos (presunto, salsicha, patês etc.) \\
\hline Batata & $\begin{array}{l}\text { Batatas novas } \\
\text { Batatas maduras, porém deixadas ao ar livre por } \\
\text { dez dias }\end{array}$ & Batatas em conserva \\
\hline Frutas & Nenhuma & Todas as frutas, frescas, sucos ou em conserva \\
\hline Hortaliças & $\begin{array}{l}\text { Vagem, abobrinha, salada verde, espinafre, aipo, } \\
\text { alho-poró, aspargos, couve-flor, agrião, endívia }\end{array}$ & $\begin{array}{l}\text { Todos os outros legumes, frescos ou em } \\
\text { conserva. Legumes secos, grão de bico, lentilhas, } \\
\text { fava, ervilhas secas. }\end{array}$ \\
\hline Condimentos & $\begin{array}{l}\text { Sal, pimenta, vinagre, gelatina, maionese caseira } \\
\text { (ovos, vinagre e óleo) }\end{array}$ & $\begin{array}{l}\text { Cebola, pepino pequeno, Ketchup, canela, } \\
\text { baunilha, molhos comerciais }\end{array}$ \\
\hline Açúcares & $\begin{array}{l}\text { Glicose, maltose, dextrina-maltose, cacau não açu- } \\
\text { carado, doces caseiros (com leite natural, sem } \\
\text { açúcar, farinha de trigo, ovos, manteiga, glicose), } \\
\text { cremes caseiros com glicose }\end{array}$ & $\begin{array}{l}\text { Frutose e sacarose. } \\
\text { Mel, bombons, confeitos, marmelada, castanha, } \\
\text { açúcar de cana, beterraba, chocolates e cacau } \\
\text { com açúcar }\end{array}$ \\
\hline Gordura & Manteiga, óleo, margarina, toucinho, banha & Nenhuma \\
\hline Bebidas & Chá, café, água mineral, infusões & $\begin{array}{l}\text { Bebidas lácteas com açúcar, limonadas, sodas, } \\
\text { xaropes de frutas, cidras, sucos de frutas, } \\
\text { achocolatados. }\end{array}$ \\
\hline Diversos & Produtos com sorbitol & Medicamentos com açúcar, agar, goma arábica \\
\hline Leites infantis & Leite materno, fórmulas sem sacarose & \\
\hline Substitutos do leite & Alfaré, Pregestimil, pregomine & \\
\hline
\end{tabular}

Fonte: Carlier \& Le Bars ${ }^{32}$ 
da infusão intravenosa de frutose, que provocará hipoglicemia, queda acentuada e prolongada no fosfato inorgânico plasmático, alterações urinárias com aumento do pH urinário e excreção de fosfato nos pacientes com intolerância hereditária à frutose. Essas alterações são reversíveis após o teste. A biópsia hepática para a determinação da atividade da aldolase é a etapa seguinte da investigação diagnóstica.

O tratamento consiste na introdução de dieta isenta de frutose (Quadro 2), o que permitirá uma evolução adequada. Um aspecto interessante é que pacientes adultos com intolerância hereditária à frutose não apresentam cárie dentária ${ }^{30}$.

\section{Deficiência de frutose 1,6-difosfatase}

Baker e Winegrad identificaram, em 1970, pela primeira vez, a deficiência de frutose-1,6-difosfatase em uma menina com cinco anos de idade, com episódios repetidos de acidose metabólica e hipoglicemia desde os seis meses de idade ${ }^{30}$. O defeito básico é a deficiência da enzima frutose-1,6-difosfatase, sendo que o fígado só poderá produzir glicose a partir do glicogênio enquanto perdurarem suas reservas. A frutose-1,6-difosfatase é necessária para a condensação das trioses em frutose-1,6-difosfato e, portanto, para a formação de glicose a partir de outras fontes que não a glicose ${ }^{31}$.

Os pacientes com deficiência de frutose-1,6-difosfatase apresentam episódios graves de hipoglicemia e acidose metabólica no período neonatal ou nos primeiros meses de vida. Tais episódios são desencadeados pelo jejum prolongado ou infecções febris, e são acompanhados por anorexia e vômitos. Ao contrário dos pacientes com intolerância hereditária à frutose, esses pacientes toleram quantidades normais de frutose e sacarose em suas dietas e não desenvolvem aversão aos alimentos doces. Os achados laboratoriais durante as crises revelam hipoglicemia, com níveis de glicose sangüínea geralmente abaixo de $40 \mathrm{mg} / \mathrm{dL}$ e acúmulo de ácido láctico até 20mM/L e pH abaixo de 7,1. A alanina, as cetonas e o ácido úrico encontram-se elevados no sangue e na urina, e podem ser detectados também glicerol e glicerol-3-fosfato. Trata-se de doença transmitida geneticamente através de herança autossômica recessiva, atingindo mais meninas do que meninos $(1,5: 1)$.

O tratamento nas crises de hipoglicemia e acidose é feito por meio da infusão de glicose e bicarbonato. A terapia de manutenção consiste em evitar o jejum prolongado e o fornecimento de dieta adequada durante os episódios febris. Apesar de não ser necessária a eliminação da frutose e da sacarose da dieta, a redução de tais componentes é recomendada até o estabelecimento da sensibilidade de cada paciente. O álcool, em decorrência de seu poder inibitório na gliconeogênese, deve ser restringido. Após o diagnóstico da deficiência da frutose-1,6-difosfatase e o tratamento adequado ser instituído, a evolução geralmente é boa. O crescimento e o desenvolvimento são normais e a tolerância ao jejum melhora com a idade ${ }^{30}$.

\section{CONSIDERAÇÕ ES FINAIS}

Como se pode observar, a incidência dos erros inatos do metabolismo é baixa, porém o seu diagnóstico deve ser feito precocemente para evitar os danos de uma hipoglicemia persistente. Entretanto, é importante abordar outros aspectos do metabolismo da frutose, já que está havendo aumento do seu consumo pelo maior emprego da mesma em produtos industrializados. A frutose é importante precursora de lipídios, que constituem problema de saúde pública, pois são importantes na patogenia de doenças cardiovasculares. O aparecimento dos efeitos colaterais associados ao uso de frutose, mesmo a proveniente da dieta, deve servir de alerta para os profissionais da saúde e a população em geral para a necessidade do uso mais controlado. A ingestão de frutose, especialmente aquela presente nos alimentos industrializados, deve ser feita com cuidado, sempre sob a supervisão de um profissional da saúde. 


\section{REFERÊ NCIAS}

1. Wang YM, Van Eys J. Nutritional significance of fructose and sugar alcohols. Ann Rev Nutr. 1981; 1:437-75.

2. Davies PM, Smmonds HA, Singer B, Mant TG, Allen $E M$, Vassos $A B$, et al. Plasma uridine as well as uric acid is elevated following fructose loading. Adv Exp Med Biol. 1998; 431:31-5.

3. Malta O, Guimarães AP. Açúcar. In: Houais A. Enciclopédia Mirador Internacional. Rio de Janeiro: Encyclopaedia Britannica do Brasil Publicações; 1976. p.93-7.

4. Nelson KY, Lehninger AL, Cox MM. Princípios de bioquímica. 3.ed. São Paulo: Sarvier; 2000.

5. Rumessen JJ. Fructose and related food carbohydrates. Scand J Gastroenterol. 1992; 27(10):819-28.

6. Matthews RH, Pehrsson PR, Farhat-Sabet M. Sugar content of selected foods: Individuals and total sugars. In: Home Economics Research Report. Washington (DC): Hum Nutr Information Service. 1987; 48:3-14.

7. Hallfrisch J. Metabolic effects of dietary fructose. FASEB J. 1990; 4(9):2652-60.

8. Instituto Brasileiro de Geografia e Estatística. Pesquisa de orçamentos familiares [Internet] 1996. [acesso em 2002 ago 16]. Disponível em: URL: http://www.ibge.gov.br/

9. Monteiro CA, Mondini I, Costa RBL. Mudanças na composição e adequação nutricional da dieta familiar nas áreas metropolitanas do Brasil (1988-1996). Rev Saúde Pública. 2000; 34(3): 251-8.

10. Perman JA. Digestion and absorption of fruit juice carbohydrates. J Am Coll Nutr. 1996; 15(5 Suppl): S12-7.

11. Buchs $A E$, Sasson $S$, Joost $H G$, Cerasi $E$. Characterization of GLUT5 domains responsible for fructose transport. Endocrinology. 1998; 139(3):827-31.

12. Shi X, Schecll HP, Summers RM, Lambert GP, Chang RT, Xia $T$, et al. Fructose transport mechanism in humans. Gastroenterology. 1997; 113(4):1171-9.

13. Hoekstra JH, Van den Aker JHL. Facilitating effect of amino acids on fructose and sorbitol absorption in children. J Pediatr Gastroenterol Nutr. 1996; 23(2):118-24.

14. Van Schaftingen E. The discovery and role of fructose-2,6-bisphosphatate. Acta Gastroenterol Belg. 1988; 51(2):141-6.

15. Dirlewanger $M$, Schneiter $P$, Jéquier $E$, Tappy $L$. Effects of fructose on hepatic glucose metabolism in humans. Am J Physiol Endocrinol Metab. 2000; 279(4):E907-11.

16. Moore MC, Cherrington AD, Mann SL, Davis SN. Acute fructose administration decreases the glycemic response to an oral glucose tolerance test in normal adults. J Clin Endocrinol Metab. 2001; 85(12):4515-9.

17. Reiser S, Powell AS, Scholfield DJ, Panda P. Daylong glucose, insulin, and fructose responses of hyperinsulinemic and nohyperinsulinemic men adapted to diets containing either fructose or highamylose cornstarch. Am J Clin Nutr. 1989; 50(5): 1008-14.

18. Sparks JW. Galactose and fructose. In: Polin RA, Fox WW. Fetal and neonatal physiology. Philadelphia: Saunders; 1992. p.384-90.

19. Franz MJ, Bantle JP, Beebe CA, Brunzell JD, Chiasson JL, Garg A, et al. Evidence-based nutrition principles and recommendations for the treatment and prevention of diabetes and related complications. Diabetes Care. 2002; 25(1):148-98.

20. American Association of Clinical Endocrinologists. The American Association of Clinical Endocrinologists Medical Guidelines for the Management of Diabetes Mellitus: The AACE system of intensive diabetes self-management 2002 up date. Endocr Pract. 2002; 8 Suppl1: S45-7.

21. McCann ML, Chen CH, Katibak EB, Kotchen JM, Likely BF, Schwartz R. Effects of fructose in infants of diabetic mothers. N Engl J Med. 1966; 275(1): $1-7$.

22. Clark JH, Bullock L, Fitzgerald JF. Dietary fructose in the management of intractable diarrhea of infancy. J Pediatr Gastroenterol Nutr. 1986; 5(1):81-6.

23. Coarse JF, Cardoni AA. Use of fructose in the treatment of acute alcoholic intoxication. Am J Hosp Pharm. 1975; 32(5):518-9.

24. Roig T, Bartrons R, Bermúdez J. Exogenous fructose-1,6-biphosphate reduces $\mathrm{K}^{+}$permeability in isolated hepatocytes. Am J Physiol. 1997; 273 (2 Pt 1):C473-8.

25. Sherman MP. Interventions for perinatal hypoxic-ischemic encephalopathy (letter). Pediatrics. 1998; 102(3 pt 1):662.

26. Pitkänen $E$, Kanninen T. Determination of mannose and fructose in human plasma using deuterium labelling and gas chromatography/mass spectrometry. Biol Mass Spectrom. 1994; 23(9):590-5.

27. Shah SW, Zhao H, Low SY, McArdle HJ, Hundal HS. Characterization of glucose transport and glucose transporters in the human choriocharcinoma cell line, bewo. Placenta. 1999; 20(8):651-9. 
28. Bossolan G, Barreiros RC, Ferreira NM, Miranda AFM, Trindade CEP. Galactose and fructose levels in mothers and full-term newborn infants. Poster presentation - Pediatric Academic Societies and the American Academy of Pediatrics joint meeting - Boston. Pediatr Res. 2000; 47:283A.

29. Barreiros RC. Determinação dos níveis sangüíneos de frutose em recém-nascidos de termo com pesos adequados para a idade gestacional com 48 horas de vida [tese]. Botucatu: Universidade Estatual Paulista; 2001.
30. Van den Berghe G. Inborn errors of fructose metabolism. Annu Rev Nutr. 1994; 14:41-58.

31. Froesch ER. Disorders of fructose metabolism. Clin Endocrinol Metab. 1976; 5(3):599-611.

32. Carlier L, Le Bars MA. Régime des intolerérances aux sucres. In: Encyclopedie Médico-Chirurgicale. Paris: Editions Techniques; 1992. Pédiatrie, $4002 \mathrm{H}^{40}$.

Recebido para publicação em 23 de setembro de 2002 e aceito em 3 de março de 2004. 